\title{
Three-dimensional Nanostructuring of Transparent Materials by the Femtosecond Laser Irradiation
}

ARTICLE in JOURNAL OF LASER MICRO / NANOENGINEERING · DECEMBER 2006

Impact Factor: 1.01 · DOI: 10.2961/jImn.2006.03.0006

CITATIONS

8

1 AUTHOR:

Yasuhiko Shimotsuma

Kyoto University

153 PUBLICATIONS 1,684 CITATIONS
READS

14

SEE PROFILE 


\title{
Three-dimensional Nanostructuring of Transparent Materials by the Femtosecond Laser Irradiation
}

\author{
Y. Shimotsuma ${ }^{*}$, M. Sakakura ${ }^{*}$, S. Kanehira ${ }^{*}$ J. Qiu ${ }^{* *}$, P. G. Kazansky ${ }^{* * *}$, K. Miura ${ }^{*}$ K. Fujita ${ }^{*}$, and K. Hirao* \\ * Department of Material Chemistry, Kyoto University, Kyotodaigaku-Katsura, Nishikyo-ku, Kyoto \\ 615-8510, Japan \\ E-mail: yshimo@collon1.kuic.kyoto-u.ac.jp \\ ** Department of Materials Science and Engineering, Zhejiang University, Hangzhou, Zhejiang \\ Province 310027, China \\ Optoelectronics Research Centre, University of Southampton \\ SO17 1BJ, United Kingdom
}

\begin{abstract}
Femtosecond laser is a perfect laser source for materials processing when high accuracy and small structure size are required. Due to the ultra short interaction time and the high peak power, the process is generally characterized by the absence of heat diffusion and, consequently molten layers. Various induced structures have been observed inside glasses after the femtosecond laser irradiation. Here, we report the refractive index change, space-selective valence state manipulation of active ions and precipitation control of nanoparticles by a femtosecond laser in glasses. We have recently observed the three-dimensional nanostructuring such as a nano-grating and a nano-void inside a glass material by the single laser beam irradiation. The self-organized sub-wavelength periodic nanostructures are created by via a pattern of interference between the incident light field and the electric field of the bulk electron plasma wave. The mechanisms of the observed phenomena were also discussed. Furthermore, we observed the space-selective structural-phase transformation from diamond to amorphous structure which has electrically conductive property.
\end{abstract}

DOI: $10.2961 / \mathrm{jlmn} .2006 .03 .0006$

Keywords: nanostructure, plasma, interference, diamond, amorphous

\section{Introduction}

A progress in high power ultrashort pulse lasers has opened new frontiers in physics and technology of lightmatter interactions. Various structures can be produced at the focal point inside a transparent material by using pulsed laser operating at the non-resonant wavelength with pulse widths of the order of femtoseconds: colored line due to the formation of color center, refractive index spot due to densification and defect formation, microvoid due to remelting and shock wave, microcrack due to destructive breakdown etc. We have demonstrated the observed induced microstructures can be used for three-dimensional marking [1], fabrication of three-dimensional optical memory with ultrahigh storage density [2-3], optical circuit [4-6], microoptical elements [7] and photonic nanostructures [8,9].

In this paper, we review our recent research developments on the femtosecond laser induced three-dimensional nanostructures and their applications.

\section{Photoinduced self-organized nanostructures}

We used commercially available synthetic silica glass, ED-H (Tosoh Quartz Corp., brands of wet silica with $\mathrm{OH}$ $50 \mathrm{ppm}$ ) of $10 \mathrm{~mm} \times 10 \mathrm{~mm} \times 2 \mathrm{~mm}$ size in our experiments. The laser radiation in Gaussian mode produced by regenerative amplified mode-locked Ti:Sapphire laser (Coherent Inc.) operating at a wavelength of $800 \mathrm{~nm}$ was focused via $100 \times(\mathrm{NA}=0.95)$ microscope objective into the silica glass samples. After laser irradiation the sample was polished to the depth of the beam waist location. The surface of the polished sample was analyzed by scanning elec- tron microscope (JEOL, JSM-6700F) and Auger electron spectroscopy (PHI, SAM-680). Secondary electron (SE) images and backscattering electron (BE) images of the same surface were compared (Fig. 1). It is well known that the SE image reveals the surface morphology of a sample, while the BE image is sensitive to the atomic weight of the elements or the density of material constituting the observation surface. The SE images of the polished silica sample indicate that the morphology of an irradiated sample in the examined cross-section does not change, namely, a void does not exist. On the other hand, the BE images reveal a periodic structure of stripe-like dark regions with low density of material and of $\sim 20 \mathrm{~nm}$ width which are aligned perpendicular to the writing laser polarization direction. We speculated, based on the fact that the elements constituting the sample are silicon and oxygen (average molecular weight of $\mathrm{SiO}_{2}$ glass $\sim 60.1$ ), that the oxygen defects were formed in the regions corresponding to dark domains of the $\mathrm{BE}$ image, which reduce the average molecular weight in these regions $\left(\mathrm{SiO}_{2-x} \sim 60.1-16 x\right)$. The Auger spectra signal of the oxygen in the regions corresponding to dark domains in the BE image is lower compared to other regions, indicating low oxygen concentration in these domains. Furthermore, there is some indication that the intensity of the oxygen signal is stronger in the regions between the dark domains of the BE image. On the other hand, the intensity of the silicon signal is the same in the whole imaged region. These results indicate that the oxygen defects $\left(\mathrm{SiO}_{2-x}\right)$ are periodically distributed in the focal spot of the irradiated region. The Auger signal intensity is proportional to the 
concentration of element constituting the surface, which gives an estimate to the value $x \sim 0.4$. We have also observed a strong reflection from the modified regions occurring only along the direction of the polarization of the writing laser. This phenomenon has given the evidence of periodic, sub-wavelength grating-like distribution of the index inhomogeneities in the irradiated spot inside the glass, which could also explain the birefringence phenomenon [10]. To facilitate the measurement of the refractive index change of modified region with respect to the unprocessed bulk, we used an interferometric phase-stepping technique [11]. We found that the index variation of the selforganized nano-gratings $\Delta n$ is about $10^{-1}$ because the oxygen defect formation lead to a decrease of density in glass structure $[9,12]$. We have also observed that the period of self-organized grating structures can be controlled from 140 to $320 \mathrm{~nm}$ by the pulse energy and the number of irradiated pulses [8].
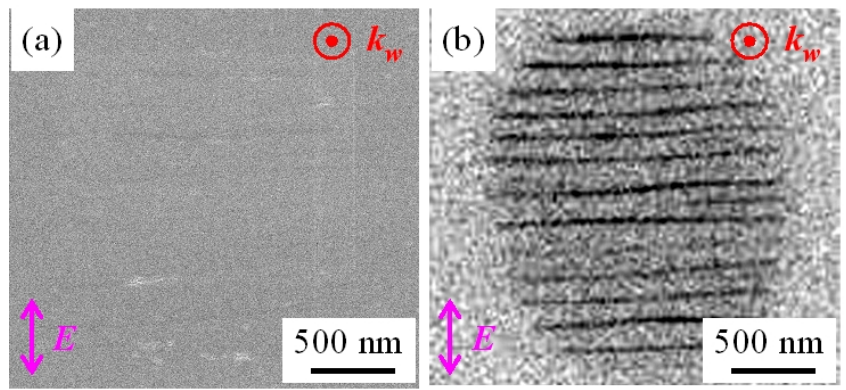

Fig. 1 (a) Secondary electron images of silica glass surface polished close to the depth of focal spot. (b) Light "fingerprints": Backscattering electron images of the same surface. $\boldsymbol{k}_{w}$ and $\boldsymbol{E}$ show the polarization direction and the wave vector of the femtosecond laser pulse, respectively.

The following explanation of the observed phenomenon is proposed. The light intensity in the focus of the beam is of $10^{14} \mathrm{~W} / \mathrm{cm}^{2}$, which is high enough for multiphoton ionization of glass matrix. Once a high free electron density is produced by multiphoton ionization the material has the properties of plasma and will absorb the laser energy via one-photon absorption mechanism of inverse Bremstrahlung heating. The light absorption in the electron plasma will excite bulk electron acoustic waves. These are longitudinal waves with the electric field component parallel to the direction of propagation. Such electron plasma wave could couple with the incident light wave only if it propagates in the plane of light polarization. Initial coupling is produced by inhomogeneities induced by electrons moving in the plane of light polarization. The coupling is increased by a periodic structure created via a pattern of interference between the incident light field and the electric field of the bulk electron acoustic wave, resulting in the periodic modulation of the electron plasma concentration and the structural changes in glass.

We also observed the cross-section of the polarizationdependent periodic nanostructure (Fig. 2). The single femtosecond laser beam with the different linear polarization directions was irradiated inside a fused silica at a $100 \mu \mathrm{m}$ under the sample surface and scanned the focal spot in the $\mathrm{X}-\mathrm{Y}$ plane perpendicular to the laser propagation. The cross-section observations were carried out on the $\mathrm{X}-\mathrm{Z}$ plane perpendicular to the scanning direction after the polishing and the subsequent wet etching of the polished sample surface. The polarization-dependent planar nanostructures are obviously aligned perpendicular to the writing laser polarization direction [Fig. 2 (c), (d)]. Furthermore, the periodic nanostructures with the period of $\sim 560 \mathrm{~nm}$ were also observed in the direction of laser propagation [Fig. 2 (a), (b)]. This period corresponds to the wavelength within the sample $(800 \mathrm{~nm} / 1.45 \sim 550 \mathrm{~nm})$. From this result, the nanostructures along with the laser propagation direction are probably formed by the interference between the two photon vectors and the two electron plasma density waves. In the case of the focusing by high NA objective lens, the polarization-dependent periodic nanostructures were formed at the region above and below the focal spot $(\sim 15 \mu \mathrm{m})$. Based on the fact that the periodic nanostructure could not be observed in the region of filamentation, the present technique will be useful in the fabrication of the polarization-dependent three-dimensional photonic devices.
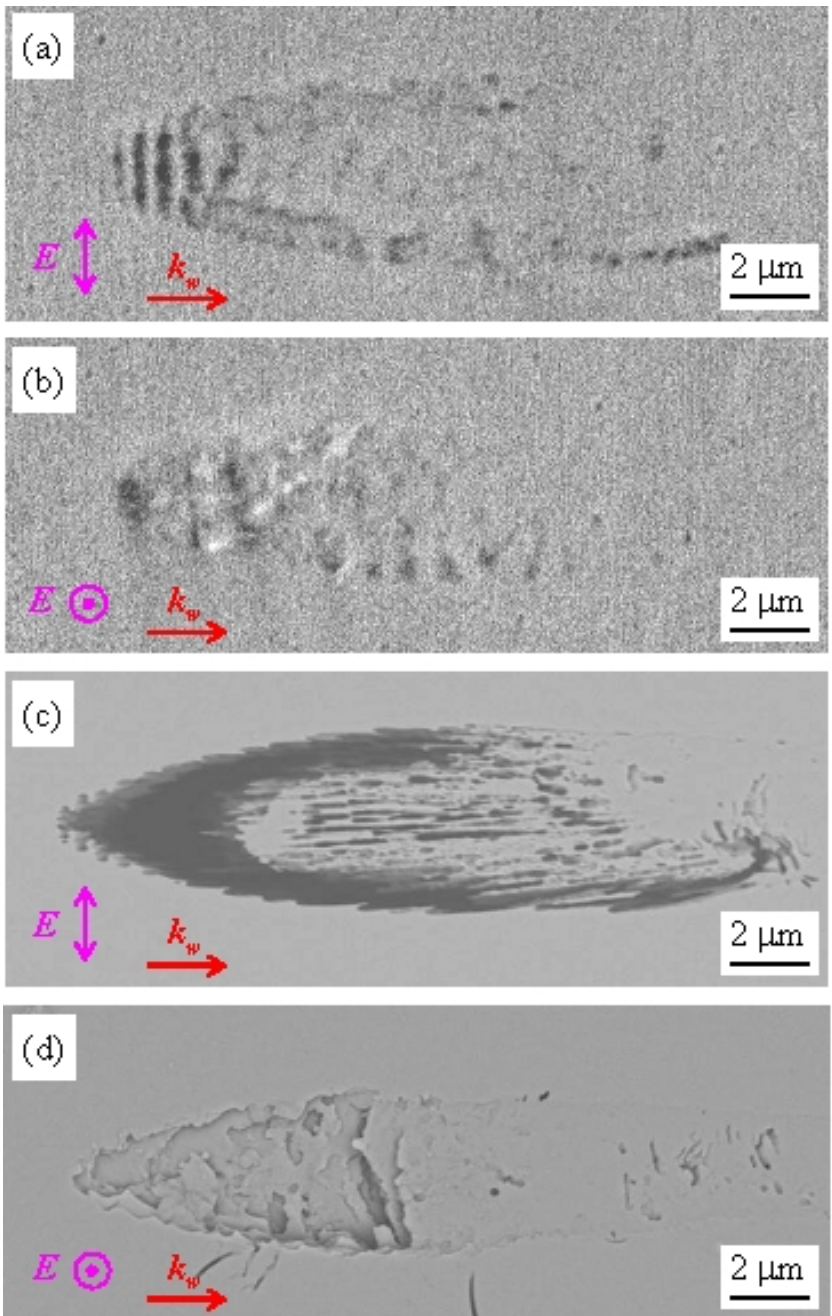

Fig. 2 Cross-sectional observation of the periodic nanostructures induced by the femtosecond single laser beam with the different linear polarization directions on the polished sample surface (a), (b) and subsequently etched surface by the hydrofluoric acid (c), (d). $\boldsymbol{k}_{w}$ and $\boldsymbol{E}$ show the laser propagating and polarization direction, respectively. 
Apart from the fundamental importance of the observed phenomenon as the first evidence of interference between light and electron acoustic waves, the observed light "fingerprints" are the smallest embedded structures ever created by light. The related phenomena of anisotropic light scattering, reflection and birefringence has been observed in various glasses and crystals. This indicates that the photoinduced three-dimensional nanostructuring is a universal phenomenon in transparent materials and could be useful for optical recording and photonic crystal fabrication.

\section{Photoinduced space-selective phase change}

We used commercially available single crystal diamond (Sumitomo Electric Hardmetal Corp., blands of Type Ib diamond with $\sim 100 \mathrm{ppm}$ ) of $3 \mathrm{~mm} \times 3 \mathrm{~mm} \times 1 \mathrm{~mm}$ size in our experiments. The laser radiation in Gaussian mode produced by regenerative amplified mode-locked Ti:sapphire laser (Cyber Laser Inc., 215 fs pulse duration, 1 $\mathrm{kHz}$ repetition rate) operating at a wavelength of $780 \mathrm{~nm}$ was focused via $20 \times(\mathrm{NA}=0.45)$ microscope objective into the diamond on the X-Y-Z stage. The beam waist diameter and laser energy fluence were estimated to be $\sim 2 \mu \mathrm{m}$ and $28.5 \mathrm{~J} / \mathrm{cm}^{2}$, respectively. The focal spot was scanned from bottom to top surface along the laser propagation direction. After laser writing, the sample surface was processed by using a focused ion beam (FIB), and then Raman spectra of the laser-irradiated regions were measured by a microraman spectrometer (Nicolet, Almega).

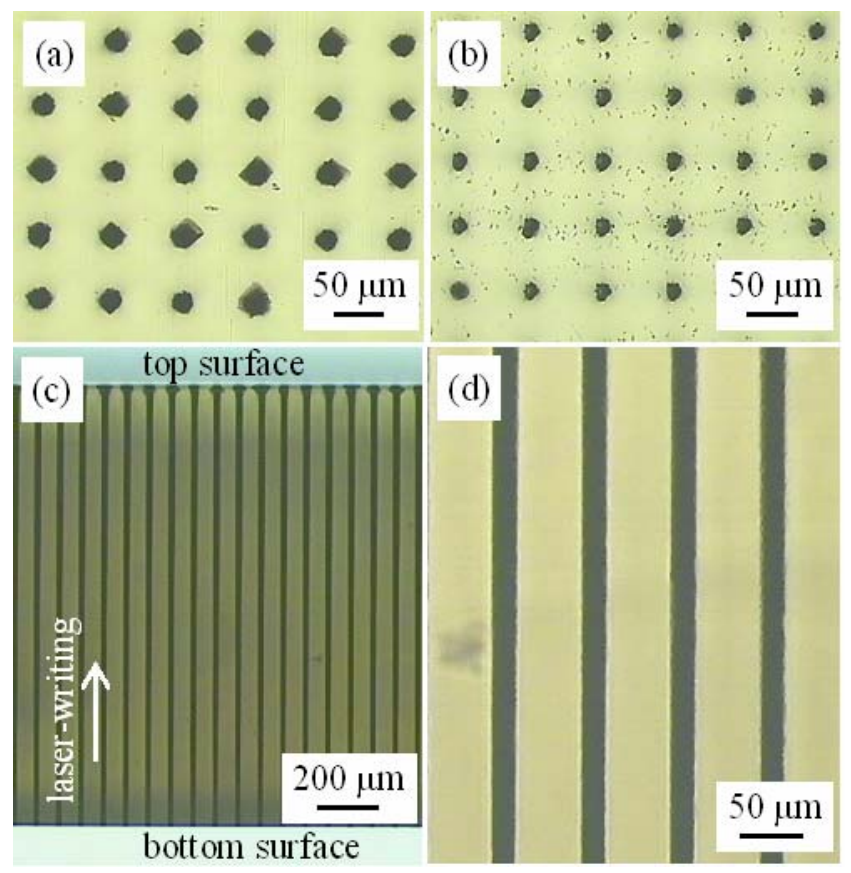

Fig. 3 Optical microscope observations of (a) top and (b) bottom surface after the laser-writing. Lateral observations are also shown in (c), (d). The magnification of the (c) and (d) images is $5 \times$ and $20 \times$, respectively. Allow shows the laserwriting direction.

After scanning the focused laser pulses with pulse energy of $1.0 \mu \mathrm{J}$ at a rate of $20 \mu \mathrm{m} / \mathrm{s}$, a black and unbroken line with a diameter of about $18 \mu \mathrm{m}$ was observed by optical microscope (Fig. 3), but in case of the laser-scanning speed of $100 \mu \mathrm{m} / \mathrm{s}$, no apparent unbroken line was ob- served. To measure the electric resistivity of the photoinduced structures by a tester, we created a $40 \times 40$ pillars from the bottom to top surface of the diamond at intervals of $70 \mu \mathrm{m}$ by scanning the focused laser pulses at a rate of 2 $\sim 50 \mu \mathrm{m} / \mathrm{s}$. Fig. 4 shows the specific resistance of a piece of photoinduced line for varying laser-scanning speed. We found that the specific resistance reaches a minimum $(\sim 1.5$ $\left.\times 10^{-2} \Omega \mathrm{m}\right)$ at scanning speed of $20 \mu \mathrm{m} / \mathrm{s}$, while the initial diamond (Type $\mathrm{Ib})$ has insulating properties $\left(\sim 10^{14} \Omega \mathrm{m}\right)$. This indicates that the optimal condition exists in transformation from diamond to amorphous structures by the femtosecond laser irradiation. Typical specific resistivity of several carbonaceous matters is shown in Table 1. From the comparison of the specific resistivity, the laser-induced unbroken pillars in diamond have conducting properties and are probably attributed to the composite material of graphite and amorphous carbon.

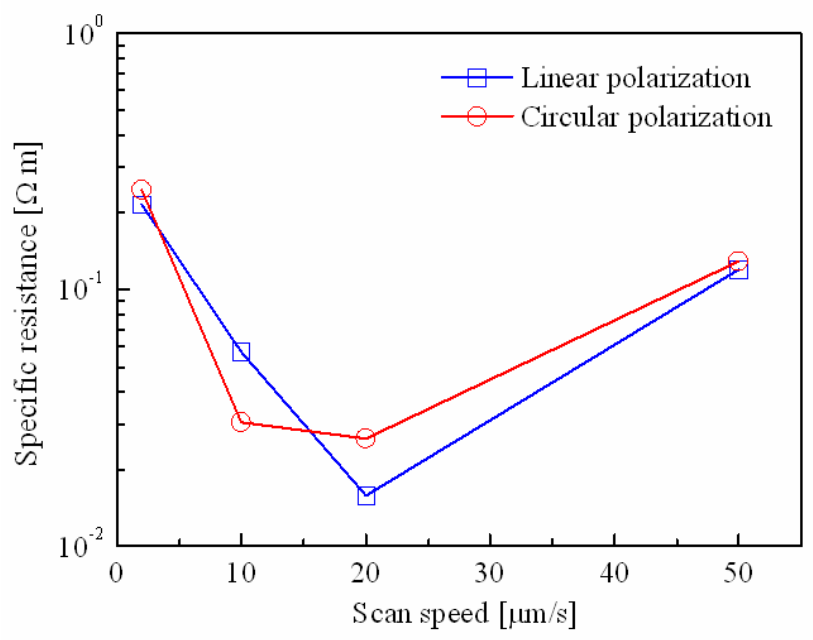

Fig. 4 Specific resistivity of a piece of photoinduced line for varying laser-scanning speed.

Table 1 Typical specific resistivity of carbonaceous matter

\begin{tabular}{l|c}
\hline \multicolumn{1}{c|}{ Material } & Specific resistivity $[\Omega \mathrm{m}]$ \\
\hline Graphite & $10^{-5} \sim 10^{-4}$ \\
\hline Amorphous carbon & $10^{3} \sim 10^{4}$ \\
\hline Diamond like carbon & $10^{4} \sim 10^{10}$ \\
\hline
\end{tabular}

Fig. 5 shows the scanning electron image of a small rectangle carved out from around laser-induced structures. From this image, the laser-induced structures are obviously densified. We have also observed the Raman spectra of the laser-induced regions (Fig. 6). Raman spectra of several reference materials and unirradiated region are also shown. From the comparison of these Raman spectra, an apparent broad peak around $1550 \mathrm{~cm}^{-1}$ was observed and assigned to $\mathrm{sp}^{2} \mathrm{C}-\mathrm{C}$ stretching vibration [13]. A sharp peak around $1330 \mathrm{~cm}-1$ attributed to diamond was also observed. We confirmed that the laser-induced regions are formed a densified composite of graphite, amorphous carbon, and diamond like carbon. The above results indicate that the photoinduced structural-phase transformation from diamond to amorphous structures which has electrically conductive property could be useful for not only the electric integrated circuit which has a high heat conductance, but also meta-material fabrication. 


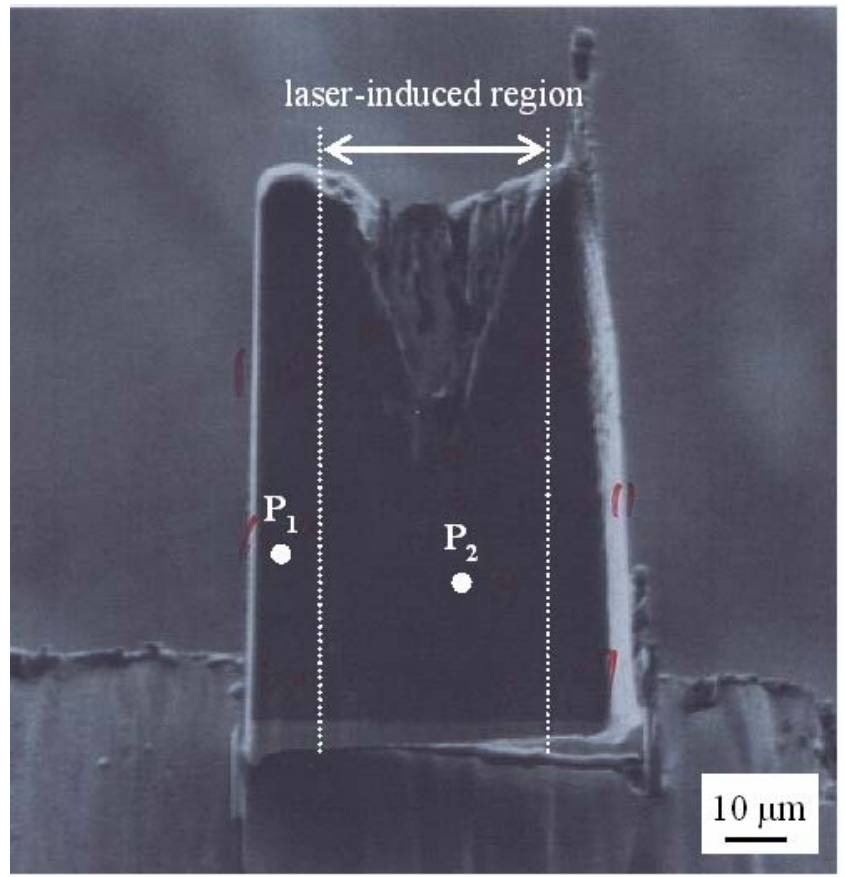

Fig. 5Scanning electron image of a small rectangle carved out from around laser-induced structures. $\mathbf{P}_{1}$ and $\mathbf{P}_{2}$ show Raman spectra measurement points.

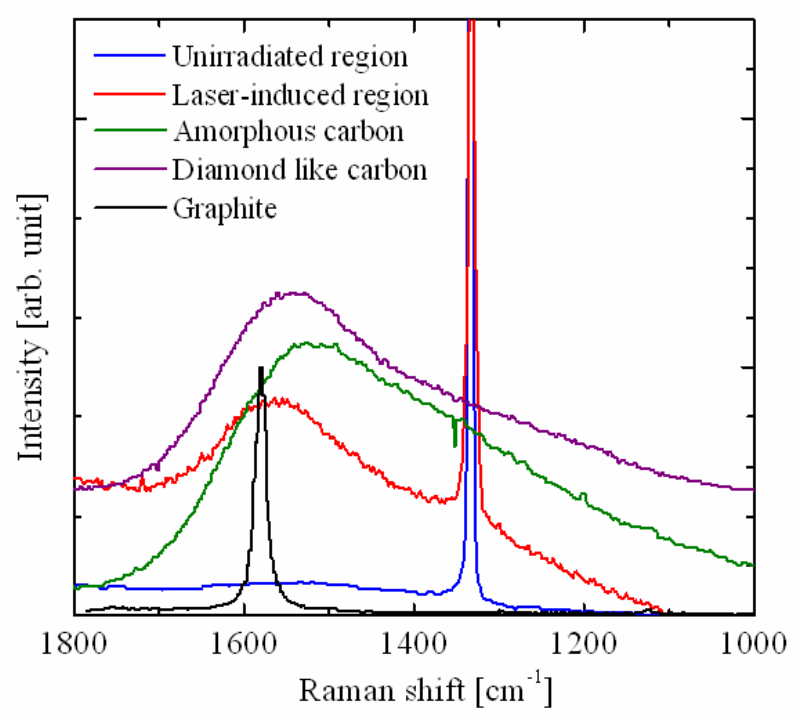

Fig. 6 Raman spectra at the point of the laser-induced regions. Raman spectra of several reference materials and unirradiated region are also shown.

\section{Conclusion}

We have observed various mysterious interactions between femtosecond laser pulses and a material relating to three-dimensional nanostructuring. We have also succeeded in the structural-phase transformation from diamond to amorphous structures which have high electrically conductive property by the femtosecond laser pulses irradiation. We have confirmed that the femtosecond laser induced nanostructures will open a new possibility in the realization of the novel physical and chemical nano-processing technologies.

\section{Acknowledgments}

This work was partially supported by Japanese Ministry of Education, Culture, Sports, Science and Technology (MEXT), and by the Mitsubishi Foundation. They are the computational materials science unit in Kyoto University, the Grant-in-Aid for Scientific Research on Priority Areas (No. 751), the Grant-in Aid for Young Scientists (B), and the 21 st century COE program.

\section{References}

[1] J. Qiu, K. Miura, and K. Hirao: Jpn. J. Appl. Phys., 37, (1998) 2263. (Journals)

[2] J. Qiu, K. Miura, T. Suzuki, T. Mitsuyu and K. Hirao: Appl. Phys. Lett., 74, (1999) 10. (Journals)

[3] Y. Shimotsuma, K. Hirao, P.G. Kazansky, and K. Hirao: Jpn. J. Appl. Phys., 44, (2005) 4735. (Journals)

[4] K. M. Davis, K. Miura, N. Sugimoto, and K. Hirao: Opt. Lett., 21, (1996) 1729. (Journals)

[5] K. Hirao, Y. Shimotsuma, J. Qiu, and K. Miura: Glass Technology, 46, (2005) 207. (Journals)

[6] K. Miura, J. Qiu, H. Inouye, T. Mitsuyu, and K. Hirao: Appl. Phys. Lett., 71, (1997) 3329. (journals)

[7] T. Nakaya, J. Qiu, K. Hirao: New Glass, 17, (2002) 32. (Journals)

[8] Y. Shimotsuma, P.G. Kazansky, J. Qiu, and K. Hirao: Phys. Rev. Lett., 91, (2003) 247405. (Journals)

[9] Y. Shimotsuma, J. Qiu, P.G. Kazansky, and K. Hirao: Mod. Phys. Lett. B, 19, (2005) 225. (Journals)

[10] J.D. Mills, P.G. Kazansky, E. Bricchi, and J.J. Baumberg: Appl. Phys. Lett. 81, (2002) 196. (Journals)

[11]C. Joenathan: Appl. Opt. 33, (1994) 4147. (Journals)

[12]E. Bricchi, B.G. Klappauf, and P.G. Kazansky: Opt. Lett., 29, (2004) 119, (Journals)

[13] Q. Wu, L. Yu, Y. Ma, Y. Liao, R. Fang, L. Zhang, X. Chen, and K. Wang: J. Appl. Phys. 93, (2003) 94. (Journals)

(Received: May 16, 2006, Accepted: October 27, 2006) 